\title{
Albert Mathiez, historien de la Révolution Française
}

Le métier d'historien face aux manipulations de l'histoire

Albert Mathiez, historian of the French Revolution. The métier of the historian confronted by the manipulations of History

\section{Florence Gauthier}

\section{(2) OpenEdition}

\section{Journals}

\section{Édition électronique}

URL : https://journals.openedition.org/ahrf/11109

DOI : 10.4000/ahrf.11109

ISSN : 1952-403X

Éditeur :

Armand Colin, Société des études robespierristes

Édition imprimée

Date de publication : 1 septembre 2008

Pagination : 95-112

ISBN : 978-2-200-92515-4

ISSN : 0003-4436

\section{Référence électronique}

Florence Gauthier, «Albert Mathiez, historien de la Révolution Française », Annales historiques de la Révolution française [En ligne], 353 | juillet-septembre 2008, mis en ligne le 01 septembre 2011, consulté le 01 juillet 2021. URL : http://journals.openedition.org/ahrf/11109 ; DOI : https://doi.org/ 10.4000/ahrf.11109

Ce document a été généré automatiquement le 1 juillet 2021.

Tous droits réservés 


\title{
Albert Mathiez, historien de la Révolution Française
}

\author{
Le métier d'historien face aux manipulations de l'histoire \\ Albert Mathiez, historian of the French Revolution. The métier of the historian \\ confronted by the manipulations of History
}

Florence Gauthier

«Que l'Europe apprenne que vous ne voulez plus
un malheureux ni un oppresseur sur le territoire
français ; que cet exemple fructifie sur la terre;
qu'il y propage l'amour des vertus et le bonheur.
Le bonheur est une idée neuve en Europe !
Saint-Just, Décrets de ventôse, rapport du 3 mars

1794.

1 Albert Mathiez, le célèbre historien de la Révolution française, fondateur de la Société des études robespierristes, en novembre 1907, et de la revue Annales révolutionnaires en 1908, demeure néanmoins un penseur méconnu et encore insuffisamment étudié.

2 Bien sûr, on remerciera James Friguglietti ${ }^{1}$, ici présent, d'avoir publié la première, et fort utile, biographie d'Albert Mathiez, en 1974, mais la matière comme le temps écoulé, invitent à approfondir notre connaissance d'un des grands historiens du XX siècle, qui rencontra, malgré sa mort prématurée, une audience internationale ${ }^{2}$.

3 Mon modeste apport se limitera à tenter de dégager les raisons du combat acharné que mena Mathiez pour faire admettre le droit de penser librement et l'indépendance de la recherche, en l'occurrence en histoire de la Révolution française. Le contexte historique de ce rude combat, celui de la Première Guerre mondiale et de la Révolution russe, était particulièrement lourd et plein de dangers. 


\section{Bref rappel de la vie d'Albert Mathiez}

4 Commençons par un bref rappel des faits marquants de la vie d'Albert Mathiez avant d'en venir à son métier d'historien. Albert Mathiez est né le 10 janvier 1874, en Franche-Comté, de parents paysans. Il fit de brillantes études. Malheureusement pour lui, durant son service militaire en 1893, il perdit l'œil gauche. L'année suivante, il fut admis à l'École normale supérieure, où il retrouva ses amis Charles Péguy et Albert Lévy, avec lesquels il partagea la défense de Dreyfus ${ }^{3}$.

5 En 1900, il prépara une thèse d'histoire sous la direction d'Alphonse Aulard, professeur à la Sorbonne occupant la fameuse chaire d'Histoire de la Révolution française. La soutenance de sa thèse, La Philanthropie et le culte décadaire, 1796-1802, eut lieu en 1903 et celle de sa thèse complémentaire, Les origines des cultes révolutionnaires, 1789-1792, l'année suivante.

6 En 1902, Mathiez épousa Marguerite Boudot, dont il divorça en 1912, après avoir eu quatre enfants.

7 En 1907, Mathiez fondait la Société des études robespierristes, dont le siège se trouvait chez l'éditeur Leroux, 28 rue Bonaparte à Paris, et publiait le premier numéro des Annales révolutionnaires en 1908. Puis, en 1913 paraissait le premier tome des Euvres de Robespierre ${ }^{4}$, ce qui correspondait à un des objectifs que s'était fixés la nouvelle société.

8 Aux tous débuts de la guerre, en septembre 1914, son ami Charles Péguy trouvait la mort au combat.

Albert Mathiez écrivit des articles sur la façon de gouverner le pays en période de guerre, sur la situation de l'Europe et du monde, parallèlement à la publication de travaux consacrés à l'histoire de la Révolution, sous forme de livres, car la revue fut interrompue en 1915.

10 En 1917, à l'annonce de la Révolution russe, la Société des études robespierristes envoya des félicitations à la Douma et, quelques mois plus tard, Mathiez prit la défense de la prise du pouvoir par les Bolcheviks.

11 Il se remaria en 1918 et obtint, l'année suivante, un poste à l'Université de Dijon's En 1920, il adhéra au Parti communiste, qui venait juste de se créer, et publia cette même année deux articles comparant les Révolutions française et russe: "Bolchévisme et jacobinisme » et «Lénine et Robespierre $»^{6}$. En 1921 et 1922, il publia, dans L'Humanité devenue l'organe du Parti communiste et dans L'Internationale, des articles sur la Révolution française ainsi que sur la politique française, parfois sous le pseudonyme de Memor. Durant toute la période précédente, il fut un membre actif de la Ligue des droits de l'homme et continua par la suite.

En 1922, Mathiez commença à s'inquiéter des interventions de la Troisième Internationale dans la vie du Parti communiste français et de sa presse, et cessa d'écrire dans ses journaux à partir du mois de septembre de la même année. La Troisième Internationale demanda l'exclusion des membres du PCF sous l'accusation suivante: " ayant des mœurs bourgeoises ». Mathiez préféra démissionner, ce qu'il fit à la fin de l'année 1922. Il était donc resté au Parti communiste deux ans.

13 En 1924, sa revue fusionna avec celle de Gustave Laurent pour devenir les Annales historiques de la Révolution Française. De 1926 à 1929, Mathiez remplaça Philippe Sagnac 
dans son poste de professeur à la Sorbonne. Il eut la douleur de perdre sa femme en 1927. Au retour de Sagnac, Mathiez obtint une charge de cours à la Sorbonne et un poste à l'École des hautes études. Ce fut une période de grand succès et de voyages à l'étranger. Les deux dernières années de sa vie furent marquées par les progrès du stalinisme en Russie et la rupture violente qu'ils entraînèrent avec les historiens soviétiques dont, pour quelques-uns, Mathiez avait publié les travaux dans sa revue.

Quand Mathiez apprit l'arrestation d'Eugène Tarlé, qu'il avait bien connu, puis l'exécution de quarante-huit intellectuels en Russie, il protesta publiquement. Il reçut en réponse, de la part des historiens soviétiques favorables à la politique stalinienne, des insultes qui dépassaient la rupture de relations, puisqu'il s'agissait d'une véritable condamnation: en 1930, Albert Mathiez était devenu «l'ennemi de la révolution prolétarienne ". Tout ceci ne l'empêcha pas de répondre à ses accusateurs durant le peu de temps qui lui restait à vivre, car en effet, le 25 février 1932, Mathiez succombait, en plein cours, à une hémorragie cérébrale, à l'âge de 58 ans.

\section{Trois moments significatifs dans la vie intellectuelle d'Albert Mathiez}

15 Ces trois moments furent sa rencontre, suivie de sa rupture, avec Alphonse Aulard, l'épreuve de la Guerre de 14 et enfin l'événement de la Révolution russe, qu'il suivit avec un immense intérêt et une remarquable lucidité.

\section{Les rapports de Mathiez avec Aulard}

Ce fut avec son directeur de recherche, Alphonse Aulard, que Mathiez fit ses premières armes comme historien. Mathiez a précisé lui-même que les raisons de son conflit avec Aulard étaient apparues au moment de sa préparation de thèse, dans la période 1900-1904, et pour des raisons scientifiques. Ce fut en répondant à la question de ses rapports avec Aulard, que son ami Louis Gottschalk lui avait posée très directement, qu'il fit cette réponse. Aulard qui s'apprêtait à publier un article de Mathiez dans sa revue Révolution française, lui avait demandé la chose suivante :

« Il m'avait demandé pour l'étude que je publiais dans sa revue sur les divisions du clergé réfractaire, des modifications que je refusais d'accorder. Il aurait voulu que je m'abstienne de dire dans cet article que le Directoire avait persécuté les catholiques. [...] Si j'avais le temps et l'espace, je vous expliquerais pourquoi l'histoire telle que la comprend Aulard est une histoire polémique au profit d'un parti et pourquoi je me suis élevé peu à peu, en m'affranchissant de son influence, à une vue plus objective des choses (ce que j'ai essayé de réaliser dans mon manuel). Je gênais ainsi la politique de défense républicaine d'Aulard et de ses amis radicaux qui, systématiquement, ne voulaient pas attirer l'attention sur le rôle primordial de la bourgeoisie et sur la misère du peuple qui a joué le rôle de dupe. Autrement dit, j'ai quitté de bonne heure les thèses apologétiques du maître, dès que je me suis aperçu où elles menaient $[. . .]^{7}$ ».

Ce fut donc une manipulation de l'histoire, demandée par son professeur, que Mathiez, alors jeune étudiant - il avait vingt-six ans en 1900 - refusa en toute conscience de commettre. Dans les époques de corruption banalisée, comme la nôtre, on pourra le traiter de ridicule et ajouter que l'on voit cela partout et tout le temps. Eh bien! justement, c'est ce qui, pour lui, Mathiez, établissait une distinction entre deux 
conceptions de la recherche, du savoir, de la politique, de l'éthique et de bien d'autres choses encore, qui font ou défont la dignité d'un être humain, et en l'occurrence, la valeur du travail d'un historien.

Ce fut encore pour ces mêmes raisons que Mathiez créa, avec Charles Vellay, la Société des études robespierristes en 1907 qui précisait ses objectifs de la manière suivante, il n'est pas inutile de les rappeler :

«Elle [la Société] a pour but de rechercher, de classer et de publier tous les documents historiques qui peuvent apporter, dans la biographie de Robespierre, dans l'étude de ses idées politiques, dans l'histoire de son influence, une nouvelle clarté. Elle se propose de travailler par les méthodes les plus rigoureuses et les plus précises, dans une impartialité absolue, à l'analyse d'une époque qui, défigurée par la passion, demeure encore, sur bien des points, mal étudiée, mal connue, mal jugée. Si elle considère Robespierre comme celui qui, depuis l'ouverture des États généraux jusqu'au 9 thermidor, incarne le plus parfaitement la Révolution ellemême, elle étend par une conséquence naturelle, le champ de ses investigations jusqu'à la Révolution tout entière, et jusqu'aux manifestations qui, au cours du XIX ${ }^{e}$ siècle, ont marqué le développement et l'histoire de la pensée révolutionnaire ${ }^{8}$ ».

Le triple objet est donc d'approfondir les connaissances de la personne de Robespierre, de l'événement Révolution et des développements et de l'histoire de la pensée révolutionnaire réalisés depuis, selon une méthode critique des sources comme des lignes d'interprétation, dans le refus d'une histoire manipulée, devenue la servante d'une cause. Car enfin, le savoir et la compréhension des choses humaines, comme l'histoire, ont toujours été menacés d'instrumentalisation au service de "l'action", comme le croyaient, et le voulaient, les positivistes, comme le croient ceux qui, aujourd'hui comme hier, veulent soumettre leur discipline au service de la puissance présente.

Que l'on nous permette de citer Marc Bloch à ce sujet, lorsqu'il insiste sur le caractère « jeune » de la connaissance rationnelle sortie, non sans douleur comme on le sait, pour nous limiter à l'époque moderne, d'un mode de pensée dogmatique et, comme tel, théologique, et rencontre ici la même critique formulée par Mathiez :

«Car l'histoire n'est pas seulement une science en marche. C'est aussi une science dans l'enfance : comme toutes celles qui, pour objet, ont l'esprit humain, ce tardvenu dans le champ de la connaissance rationnelle. Ou, pour mieux dire, vieille sous la forme embryonnaire du récit, longtemps encombrée de fictions, plus longtemps encore attachée aux événements les plus immédiatement saisissables, elle reste, comme entreprise raisonnée d'analyse, toute jeune. Elle peine à pénétrer, enfin, audessous des faits de surface, à rejeter, après les séductions de la légende ou de la rhétorique, les poisons, aujourd'hui plus dangereux, de la routine érudite et de l'empirisme, déguisé en sens commun $»^{9}$.

Il nous a semblé nécessaire de rappeler avec insistance que la question de la séparation d'avec Aulard a été voulue, de façon délibérée, par Mathiez, pour des raisons scientifiques touchant à la méthode historique et à la conception de l'histoire.

Le travail d'historien de Mathiez a-t-il porté ses fruits? Lui-même estimait, quelques quatorze ans plus tard, que oui et il insistait à nouveau sur la question de la méthode, seul moyen de répondre aux attaques idéologiques de tous bords :

«La société historique que j'ai l'honneur de présider depuis quatorze ans s'est donné pour tâche de laver l'histoire des Robespierristes de toutes les légendes thermidoriennes qui l'ont dénaturée. La revue qu'elle édite, les publications qu'elle patronne ont fait bonne justice de la plupart. Nos démonstrations, toutes fondées sur l'érudition la plus solide, n'ont pas jusqu'ici trouvé de contradicteurs. Je puis 
dire que, devant les savants et devant les esprits impartiaux, nous avons gagné notre procès ${ }^{10} »$.

23 Au début des années vingt, Mathiez avait effectivement réussi à se faire reconnaître sur le plan scientifique et le nombre d'adhérents à sa société dépassait, maintenant, celui de la revue d'Aulard ${ }^{11}$.

\section{Le second moment fut celui de la Guerre de 1914-1918}

Il écrivit durant la guerre de nombreux articles sous son nom, puis parfois sous un pseudonyme pour se protéger de la censure, afin d'analyser librement les politiques menées par le gouvernement français, ainsi que la situation internationale.

Dans ses travaux sur la Révolution datant de cette période, Mathiez rappelle fréquemment les enseignements du passé pour éclairer le présent et vice versa. Et ce fut par ce travail de comparaison dans la façon de mener une guerre défensive qu'il découvrit, ou plus exactement redécouvrit, le caractère démocratique de la politique menée par la Convention montagnarde, alors que le gouvernement français, en 1914-1918, menait une politique qu'il qualifie de « despotique ».

Mathiez comprit que cette expérience allait contribuer au renouvellement de l'histoire de la Révolution, sur tous les plans : politique démocratique menée par la Convention montagnarde, assemblée élue, et non confiscation du législatif au moyen du renforcement de l'exécutif, en profitant de l'état de guerre; appui sur les initiatives populaires; rôle des savants; problèmes de la vie chère qui appelèrent une politique capable de réajuster les rapports salaires, prix et profits.

Mathiez a pu approfondir, à cette occasion, un des points d'opposition entre Gironde et Montagne, la Gironde cherchant à mener une guerre de conquête européenne, la Montagne défendant le principe du droit naturel des peuples à leur souveraineté et à leur indépendance.

Il mettait ainsi, en pleine lumière, ce caractère essentiel de la politique du Comité de Salut public de l'an II, qui refusa clairement et publiquement la guerre de conquête et les annexions et prit la défense des droits des peuples à leur souveraineté.

Il nous semble intéressant de rappeler ici ce remarquable passage, qui n'a, précisons-le, pas encore été approfondi en ce qui concerne l'exemple de la Catalogne, par les successeurs de Mathiez :

«Cependant le Comité (de Salut public) n'est pas animé de l'esprit de conquête. Il veut que la guerre nourrisse la guerre, mais il ne songe pas à annexer les pays occupés. "Nous marchons, non pour conquérir, mais pour vaincre, avait dit Billaud le $1^{\text {er }}$ floréal ${ }^{12}$, non pour nous laisser entraîner par l'ivresse des triomphes, mais pour cesser de frapper à l'instant où la mort d'un soldat ennemi serait inutile à la liberté." Le comité ne veut pas perdre la République dans le militarisme. Quand Milhaud et Soubrany lui proposent de conquérir la Catalogne et de l'annexer à la France, Couthon leur répond, le 7 prairial (26 mai 1794): "Il nous paraît plus conforme à nos intérêts et à nos principes d'essayer de faire de la Catalogne une petite république indépendante qui, sous la protection de la France, nous servira de barrière à cet endroit où les Pyrénées cessent. Ce système flatterait sans doute les Catalans et ils l'adopteront plus volontiers encore que la réunion à la France. Vous devez dans les montagnes porter nos limites jusqu'aux extrémités et par conséquent vous établir à demeure dans toute la Cerdagne, prendre la vallée d'Aran, en un mot tout ce qui est en deçà des monts [...] Mais la Catalogne, devenue 
département français, serait aussi difficile à conserver que l'est aujourd'hui l'ancien

Rous-sillon $»^{13}$. français, en 1920, Mathiez y adhéra, pour en ressortir très vite, à la fin de l'année 1922. Que s'était-il passé ? Du point de vue d'Albert Mathiez, trois éléments s'imposent à l'attention.

-a. La définition du type de la Révolution russe.

-b. Le problème de l'absence de souveraineté populaire effective et, par voie de conséquence, de démocratie en Union soviétique.

- c. L'évidence du stalinisme à partir de 1930.

\section{a) L'analyse du type de la Révolution russe}

Selon Mathiez, cette révolution était, en 1917, essentiellement agraire. Le gouvernement Kerenski ne s'était pas occupé des paysans mais les Bolcheviks, eux, l'avaient fait en redistribuant la terre aux paysans, et Mathiez avait insisté dans ses articles sur ces caractères originaux de la Révolution russe.

Cette question centrale avait d'ailleurs été posée depuis longtemps en Russie et dans le dernier tiers du XIX ${ }^{e}$ siècle, de nombreux historiens russes étudièrent les questions 
agraires de la Révolution française, rappelons pour mémoire Loutchisky, Karéiev, Kovalevski, Kropotkine. On sait aussi que Véra Zassoulitch avait entretenu une correspondance avec $\mathrm{Marx}^{17}$ lui-même, sur les potentialités révolutionnaires et démocratiques de la communauté paysanne russe, le mir avec ses biens communaux et ses usages collectifs.

Bref, Mathiez a chaudement soutenu les mesures léninistes et n'a pas hésité à dresser une comparaison entre Bolcheviks et Jacobins, entre Lénine et Robespierre en 1920.

Précisons que Lénine avait, comme Mathiez, effectué le chemin de la comparaison entre les deux révolutions et avait réfléchi à ce sujet, en particulier dans un article au titre significatif, datant de mars 1905 : «Une révolution de type de 1789 ou du type de 1848 ?» ${ }^{18}$, avec comme réponse le souhait qu'elle soit de type 1789 .

En 1928, la collectivisation forcée retira leur terre aux moujiks, ain de généraliser la concentration de grandes exploitations agricoles, et les moujiks n'eurent plus d'autre choix qu'entre un avenir... d'ouvriers agricoles ou d'ouvriers d'usine. À partir de 1930, la «dékoulakisation» s'en prit, en paroles si ce n'est dans les faits, aux couches supérieures de la paysannerie. Ce faisant, l'analyse du type de la Révolution russe de 1917 s'en trouva modifiée et le terme de "révolution prolétarienne » dans la langue des années trente, n'en vint plus à désigner que «les ouvriers ", qu'ils soient agricoles ou d'usine. Cette nouvelle définition est importante pour comprendre l'idéologie stalinienne, et nous la retrouverons.

\section{b) L'absence de souveraineté populaire et de démocratie dans la révolution russe}

39 Cette question était essentielle aux yeux de Mathiez. Et dans un de ses articles de 1920, il posa clairement la question comme l'indique le titre: «Le bolchevisme est-il antidémocratique ? $»^{19}$ Il répondait qu'effectivement, il n'y avait pas d'institutions démocratiques en Russie à cause de la guerre civile mais qu'il fallait patienter et que la paix intérieure les verrait naître bientôt. La paix revenue, rien ne vint et Mathiez comprit alors que cela... ne viendrait pas. En 1920, il avait analysé avec une remarquable lucidité le pouvoir que la Révolution bolchevique avait imposé comme: « une aristocratie d'exécutants au-dessus de la masse soi-disant souveraine ${ }^{20}$.

En effet, Mathiez n'a jamais accepté le principe d'une dictature d'un parti unique au pouvoir, ou d'un chef suprême, questions qui étaient si à la mode au tournant des XIX ${ }^{\mathrm{e}}$ et $\mathrm{XX}^{\mathrm{e}}$ siècles, à gauche comme à droite. Et l'on peut affirmer que ce fut sa connaissance de la Révolution française et de la démocratie à souveraineté populaire effective, tant espérée de 1789 à 1792, puis expérimentée de 1792 à 1794, qui lui a permis de résister à cette aberration fondée sur l'ignorance, qui emporta son époque. Et ce fut précisément sur cette question de l'absence de démocratie et de respect de la souveraineté des peuples que Mathiez rompit, en 1922, avec le Parti communiste et la Troisième Internationale.

41 Cette rupture de Mathiez avec le Parti communiste mériterait une étude spéciale qu'il n'est pas possible de faire ici mais qu'il est indispensable de souligner et d'indiquer, précisément parce qu'elle a été complètement occultée. Écoutons-le :

« Je ne conçois pas de dictature au sein du parti. La dictature n'est admissible qu'en période de catastrophe, quand on ne peut pas éviter de s'en servir. Mais c'est une folie de s'imaginer qu'on peut obtenir des hommes des sacrifices par l'obéissance passive. Des commandements donnés de haut et imposés sans discussion sont bons pour des esclaves. Des hommes libres refuseront toujours de s'y conformer. 
Discipline tant qu'on voudra, mais discipline librement consentie et comprise! Le parti français ne peut pas exécuter des ordres dont les inconvénients sautent aux yeux. La France n'est pas la Russie ! ${ }^{21}$ période où il s'apprêtait à quitter le Parti communiste français. Il ne voyait plus en eux des « hommes libres » mais des hommes ayant « une mentalité d'esclaves », résultat des pratiques dictatoriales instaurées par le Parti bolchevik en Russie et que les partis communistes affiliés à la Troisième Internationale étaient en train d'imposer à travers le monde entier.

\section{c) L'évidence du stalinisme en 1930 s'est brutalement révélée avec l'arrestation de I'historien Eugène Tarlé, que Mathiez connaissait bien, et l'exécution de quarante- huit intellectuels russes.}

refusaient de voir en Mathiez un ennemi, et les défenseurs de la version stalinienne de l'histoire devenue la seule version admise du marxisme. La campagne contre l'esprit de Mathiez en vint à réclamer qu'on en finisse avec la Révolution française, qu'on n'en parle plus que comme une affaire bourgeoise, d'un passé révolu. Ce fut ainsi que le « jacobinisme devint, en 1930, un adversaire de la révolution prolétarienne ${ }^{24}$.

Ce fut encore ainsi que la Gironde et la Montagne ne furent plus que des variantes de «la » bourgeoisie au pouvoir, que les Déclarations des droits de l'homme et du citoyen, les constitutions, les institutions républicaines n'étaient plus que des préoccupations de 
bourgeois, que la démocratie, la souveraineté populaire, la citoyenneté, la séparation des pouvoirs n'étaient que des mots creux.

On notera que cette version stalinienne de l'histoire de la Révolution française partageait un certain nombre des positions de la contre-révolution depuis $1789:$ mise à part la qualification burlesque de « bourgeois » accolée au mouvement populaire et à la politique montagnarde, que la contre-révolution qualifiait, elle, "d'anarchistes » et "d'anarchie " ${ }^{25}$, l'usage des termes péjoratifs de «jacobin», « néo jacobin», "robespierriste ", lancés comme de véritables insultes ${ }^{26}$; le souhait de faire oublier l'histoire de la Révolution française et, bien évidemment comme point central, le rejet d'une " politique de la philosophie » éclairée par les droits de l'homme et du citoyen; le rejet enfin du principe de souveraineté populaire dans l'organisation d'institutions républicaines et démocratiques. Un marxisme de droite était en train de se constituer en Union soviétique.

\section{Comment Mathiez répondit-il à cette nouvelle manipulation de l'histoire?}

Dans des notes publiées dans les Annales historiques de la Révolution française de 1931, il analysait la situation de l'histoire en Union soviétique de la manière suivante : « Dans la Russie de Staline, il n'y a plus de place pour une science libre et désintéressée, pour une science tout court. L'histoire notamment n'est plus qu'une branche de la propagande ».

En ce qui concerne ce que l'on appelle «le » marxisme, bien qu'il y en ait plusieurs ${ }^{27}$ préférons donc «les » marxismes - Mathiez s'y est intéressé tout au long de sa vie. Il a vécu à l'époque où divers courants, se prétendant tous " marxistes ", furent finalement combattus par la version stalinienne qui s'affirma comme la seule et unique bonne version.

Mathiez comprit très tôt que ces formations se constituaient en dogmes, relevant bien davantage de la théologie que d'un rationalisme laïc et d'une méthode des Lumières. Ainsi, il s'était déjà inquiété de ce que fabriquait Jules Guesde, qui, on le sait, se vantait de construire un catéchisme de " marxisme orthodoxe »- y compris un prêt à penser de l'histoire - à l'usage de la classe ouvrière, considérée comme incapable de penser ${ }^{28}$.

53 Mathiez refusait, très lucidement une fois encore, d'ériger une méthode d'investigation en dogme. Et pour éviter cet écueil, il lui paraissait indispensable de la confronter au réel. Voici ce qu'il pensait du « marxisme » en 1931 :

« C'est une hypothèse sans doute, mais une hypothèse féconde qu'il y a toujours lieu d'examiner et d'essayer de vérifier dans les faits. La méthode devient néfaste, si au lieu de se présenter pour ce qu'elle doit être, elle prétend s'ériger en vérité dogmatique et démontrée. Elle est néfaste, parce qu'elle préjuge à l'avance de la question posée. Elle fausse les faits pour les faire rentrer dans la théorie et c'est le cas de nombreux historiens soviétiques qui incorporent l'interprétation marxiste de l'histoire à l'État russe d'aujourd'hui et traitent d'hérétiques tous ceux qui doutent de leurs démonstrations d'ailleurs contradictoires $»^{29}$.

On voit ainsi qu'il ne rejette pas la méthode «marxiste », mais refuse ses déformations dogmatiques, faisant montre d'un esprit indépendant, capable de penser par lui-même, sans l'aide d'autrui ni d'un parti politique, osant même affronter débats et polémiques, bref ce que l'on est en droit d'attendre d'un intellectuel digne de ce nom ${ }^{30}$. 
Saluons la perspicacité d'Albert Mathiez, mais ajoutons qu'elle n'a pas encore été reconnue et qu'elle a même été occultée. Ainsi, on a pu entendre ces dernières années François Furet se présenter comme celui qui allait libérer l'historiographie de la Révolution française de l'orthodoxie stalinienne! On notera toutefois que Furet ne parle jamais d'Albert Mathiez ${ }^{31}$ en termes d'historien clairvoyant, critique du stalinisme à sa naissance, et bien au contraire, il le range parmi les «staliniens »! Et pourtant, la clairvoyance de Mathiez est d'autant plus précieuse qu'il s'inquiétait de l'absence d'institutions démocratiques dans la Révolution russe, dès 1920, c'est-à-dire avant même la prise de pouvoir par Staline. Furet a figé l'image d'un Mathiez en adepte quasi religieux de la révolution russe, dont le ridicule rejaillit sur lui-même tel un boomerang :

"Ce qu'il [l'historien "jacobin" selon Furet] aime dans la Révolution soviétique, c'est ce qu'avait aperçu, dès 1920, Mathiez qui n'était pas marxiste : la superposition de deux images libératrices, qui constituent le tissu de notre histoire contemporaine en religion du progrès. [...] Peu importe que l'histoire de ces dernières décennies ait apporté à cette construction des démentis auxquels elle n'aurait pas dû survivre $»^{32}$.

On le voit, F. Furet ignore que Mathiez, entré au Parti communiste en 1920, en est sorti dès 1922, qu'il a exprimé, dès 1920, ses inquiétudes constantes sur l'absence d'institutions démocratiques en Union soviétique, et qu'enfin, il fut un critique perspicace du stalinisme des années trente jusqu'à sa mort en 1932, révélant ses capacités à « démentir cette construction ».

Il est certain que l'histoire et l'historiographie de la Révolution française peuvent être une remarquable école pour forger le caractère, la liberté de penser, l'indépendance d'esprit dont a fait montre Albert Mathiez, à condition de travailler comme il l'a fait, de façon érudite, sérieuse, c'est-à-dire en prenant au sérieux son lecteur, sensible enfin pour être capable de mettre en lumière les éléments déformés, occultés, manipulés au point de devenir incompréhensibles.

On est loin, bien sûr, aux antipodes mêmes de ces théologiens qui croient dissimuler leurs dogmes, en abusant des beaux mots de "philosophie de l'histoire " pour écarter les cartons d'archives poussiéreuses qu'ils n'ont peut-être pas même l'idée d'aller ouvrir.

On peut « penser » ici, en particulier, à François Furet qui a osé affirmer, en 1979, que l'érudition ne sert finalement à rien, parce qu'elle ne saurait éclairer la compréhension d'un événement historique : «C'est que l'érudition, si elle peut être stimulée par des préoccupations empruntées au présent, ne suffit jamais à modifier la conceptualisation d'un problème ou d'un événement $»^{33}$.

60 Une affirmation de cette nature laisse pantois... Certains ont signalé que nous entrions dans une période de « défaite de l'érudition $»^{34}$.

61 Le philosophe Jean-Pierre Faye ${ }^{35}$ répondit au livre de Furet, Penser la Révolution, par un chef-d'œuvre de pédagogie, avec son Dictionnaire politique portatif en cinq mots. Avec intelligence et délicatesse, car ils se connaissaient personnellement, Faye détruisait, par l'érudition, la thèse centrale du livre de Furet, qui affirmait que la Révolution française aurait été la « matrice des totalitarismes du XX ${ }^{e}$ siècle ». Faye remporta là une grande victoire, car Furet renonça, sans fracas il est vrai, à ce dogme que, personnellement, il ne reproduisit plus dans ses livres ultérieurs mais que certains de ses émules continuent, aveugles et sourds, de répéter. 


\section{La reconnaissance d'Albert Mathiez de son vivant}

Mathiez a influencé des intellectuels qui se sont intéressés à Marx de façon non dogmatique, et au problème central de la nécessaire liaison entre démocratie et socialisme.

3 Pour mieux saisir ce problème essentiel, reportons-nous à l'étude qu'il a consacrée à Buonarroti et Robespierre et qui approfondit le rapport démocratie/socialisme sur le thème suivant : «Il n'est donc pas exagéré de dire que le socialisme français de l'époque de 1830 comme le socialisme chartiste émanent tous deux de Buonarroti et par Buonarroti de Robespierre $»^{36}$.

Mathiez fut un inspirateur pour Antonio Gramsci qui, en lisant ses travaux, renouvela sa propre réflexion sur les révolutions politiques et sociales et sur la question toujours actuelle de la démocratie sociale à souveraineté populaire effective, ou « la république démocratique » de la Révolution française, déjà redécouverte par Friedrich Engels dans sa Critique du projet de programme social-démocrate de 1891.

Arthur Rosenberg ${ }^{37}$, professeur de philosophie et spécialiste d'Aristote, en Allemagne, s'intéressa, lui aussi, à Marx de façon non dogmatique et à ce qui lui paraissait l'indispensable liaison entre le socialisme et la démocratie, que défendaient Mathiez et l'historien de la démocratie chez Jefferson, Charles A. Beard ${ }^{38}$. Rosenberg eut un itinéraire comparable à celui de Mathiez, et quitta le Parti communiste, en 1927, refusant la politique de la Troisième Internationale.

Invité en Argentine en 1929, Mathiez y resta trois mois et eut des échanges fructueux avec des historiens argentins, dont Ricardo Caillet-Bois. L'intérêt pour les travaux de Mathiez est toujours vivant en Argentine comme en témoignent les publications de Horacio Sanguinetti par exemple ${ }^{39}$.

Ces intellectuels partageaient avec Albert Mathiez, et chacun à leur manière, un impératif bien prononcé pour une démocratie socialiste, alliée à une indispensable indépendance d'esprit et une liberté de penser qu'ils ont été capables d'assumer.

Que l'on nous permette de conclure en rendant hommage à Albert Soboul. Nous aurions voulu développer la façon dont Georges Lefebvre et Albert Soboul ont essayé, à travers des périodes de censure auxquelles ils furent soumis et d'autocensure qu'ils s'infligèrent, de poursuivre l'étude des mouvements populaires des campagnes et des villes. Mais, faute de temps, limitons-nous à préciser que Soboul, dont nous sommes quelques-uns, ici, à avoir suivi l'enseignement, nous a fait le don précieux de nous transmettre, si nous voulions l'entendre, non seulement l'amour de l'histoire mais aussi la méthode d'Albert Mathiez, qui avait pourtant valu à ce dernier les foudres des historiens staliniens.

« Ma méthode, écrit Mathiez en 1928, [.] consiste toujours à présenter au lecteur un récit aussi complet que possible, fondé sur les documents les plus authentiques, préalablement interprétés et critiqués dans un esprit rigoureusement scientifique.

Je n'écris pas pour catéchiser, pour recruter des adhérents à tel ou tel parti, mais pour instruire et renseigner. Je croirais déchoir à mes propres yeux si je me préoccupais, quand je prends la plume, du parti que tireront de mes écrits les politiques du jour, en France et à l'étranger. 
Que ces hommes d'action, rouge, noire ou blanche s'efforcent d'exploiter mes livres au profit de leur cause, avec plus ou moins de bonne foi, c'est un ennui que je dois supporter avec calme.

$\mathrm{Ni}$ leurs éloges, ni leurs injures ne me feront dévier de ma route. Si l'histoire est la politique du passé, ce n'est pas une raison, au contraire, pour qu'elle devienne l'humble servante de la politique ou plutôt des politiques du présent. Elle n'a de raison d'être que si elle dit en toute indépendance ce qu'elle croit être la vérité. Tant pis pour ceux que cette vérité blesse ! Ou plutôt tant mieux, car c'est peut-être une des conditions du progrès $»^{40}$.

\section{NOTES}

1. J. Friguglietti, Albert Mathiez historien révolutionnaire, 1874-1932, Paris, 1974.

2. Signalons que Stéphanie Mailhe prépare une thèse sur Robespierre et l'historiographie. On peut lire son Mémoire de M2, Les difficultés de certains marxistes du XXe siècle à comprendre le programme économique et social de Robespierre, Université Paris7-Denis Diderot, sous la direction de F. Gauthier, 2006.

3. J. Friguglietti, op. cit., p. 22-29.

4. Robespierre, CEuvres Complètes, Première partie, Robespierre à Arras, par Eugène Déprez, Paris, Leroux, 1910, reprint Paris, 2000. Neuf volumes supplémentaires ont suivi jusqu'en 1967, le dernier, le tome XI, vient de paraître, préparé par F. Gauthier, édité par la Société des études robespierristes, 2007.

5. Friguglietti, op. cit., p. 144.

6. « Bolchévisme et jacobinisme », Scientia, XXVII, 1920 ; « Lénine et Robespierre », Floréal, 12 juin 1920.

7. Cette lettre de Mathiez, datée du 23 novembre 1930, a été communiquée par L. Gottschalk et publiée dans les AHRF, 1932, p. 218-20.

8. L'annonce de la formation de la Société parut dans la Revue critique du 9 décembre 1907, texte cité dans Friguglietti, op. cit., p. 81.

9. Marc Bloch, Apologie pour l'histoire, Paris, 1949, 1997, Introduction, p. 43. La «jeunesse» de l'histoire de la Révolution française était bien réelle, car ce fut dans le dernier tiers du XIX ${ }^{\mathrm{e}}$ siècle qu'une histoire fondée en érudition apparut: Mathiez représentait la seconde génération d'historiens travaillant sur archives.

10. Mathiez, « Napoléon ou Robespierre? ", L'Internationale, 20 avril 1921, à la une.

11. Voir Friguglietti, op. cit., p. 150.

12. Le $1^{\text {er }}$ floréal an II- 20 avril 1794, Billaud-Varenne, membre du Comité de Salut public, présentait un rapport à la Convention d'où est extraite cette citation. Voir Billaud-Varenne, Principes régénérateurs du système social, Paris, 1992, texte édité par Françoise Brunel, Introduction, p. 49.

13. Mathiez, La Révolution française, t. 2, La Gironde et la Montagne, Paris (1924) 1978, chap. XIII, p. 343. (Une coïncidence intéressante : Mathiez avait projeté de préparer une thèse de géographie sur la Catalogne en 1898 mais choisit l'histoire de la Révolution française, voir Friguglietti, op. cit., p. 38). 
14. Ibid., p.344. Sur cette problématique de la guerre de conquête voir Georges Michon, Robespierre et la guerre révolutionnaire, Paris, 1938; Florence Gauthier, Triomphe et mort du droit naturel en révolution, 1789-1795-1802, Paris, 1992, 3e partie; Marc Belissa, Fraternité universelle et intérêt national, 1713-1795, Paris, 1998, $3^{\mathrm{e}}$ partie.

15. Mathiez, sous le pseudonyme de Memor, «M. Poincaré continue», L'Internationale, 21 septembre 1921.

16. Mathiez, «Le Sénat aura-t-il toujours le dernier mot?", Le Progrès civique, $\mathrm{n}^{\circ} 272$, 1er novembre 1924, cité dans Friguglietti, op. cit., p. 184.

17. Lettre du 8 mars 1881, traduite dans Marx, Engels, Lettres sur le capital, Éditions sociales, 1964, p. 305.

18. Lénine, Cuvres, Paris-Moscou, 1964, t. 8, p. 257.

19. Mathiez, « Le bolchevisme est-il antidémocratique? », Le progrès civique, 11 septembre 1920.

20. Ibid., cité dans Friguglietti, op. cit., p. 166.

21. Mathiez, "Opinions libres », Le populaire de Bourgogne, 28 juillet 1922, cité dans Friguglietti, op. cit., p. 179.

22. Tamara Kondratieva, Bolcheviks et Jacobins, Paris, Payot, 1989, Chap. XI, p. 197.

23. Ibid., Intervention d'ouverture du congrès par son président M. N. Pokrovski, citée p. 198.

24. Ibid., p. 209.

25. Voir Marc Deleplace, L'Anarchie de Mably à Proudhon, 1750-1850, Lyon, ENS Éditions, 2000. Sur le concept de "révolution bourgeoise", voir F. Gauthier, "Critique du concept de révolution bourgeoise", Raison présente, $\mathrm{n}^{\circ} 123,1997$, p.59-72, mis en ligne sur le site revolutionfrancaise.net.

26. Mathiez reçut les honneurs de l'insulte en 1931, lorsque ses «ennemis » soviétiques inventèrent un qualificatif accusateur pour mener une campagne contre la « matiezovchtchina ». T. Kondratieva précise : «Le mot est formé du radical Mathiez, suivi du suffixe péjoratif ovchtch.», op. cit., p. 203, n. 16.

27. Entre 1917 et 1930, on peut en distinguer au moins trois, les marxismes léniniste, trotskiste et stalinien, ce dernier détenant le pouvoir menait une guerre d'extermination des deux autres.

28. Mathiez, "Les traditions de la Révolution française", L'Humanité, 28 novembre 1921. Sur Guesde, voir Marc Angenot, "Jules Guesde ou la fabrication du marxisme orthodoxe ", Actuel Marx, n² 23, 1989, p. 33-46.

29. «Choses de Russie », AHRF, 1931, p. 149-58.

30. Voir Kant, «Qu'est-ce que les Lumières ? ", 1784 : « Sapere aude! Aie le courage de te servir de ton propre entendement. Voilà la devise des Lumières ", Philosophie de l'histoire, Paris, Médiations, 1947, trad. de S. Piobetta, p. 46. Sans doute, à notre époque de mépris affiché pour l'intellect et agenouillée devant de "nouveaux maîtres à penser ", choisis par les medias pour leur nuque flexible à la corruption, ce "courage" des Lumières apparaît comme un corps qui lui est étranger.

31. François Furet, Penser la Révolution, op. cit., p. 118-22.

32. Ibid, p. 122.

33. Ibid., p. 22.

34. Olivier Betourné, Aglaïa Hartig, Penser l'historiographie de la Révolution française, Paris, 1989.

35. Jean-Pierre Faye, Dictionnaire politique portatif en cinq mots. Démagogie, Terreur, Tolérance, Répression, Violence, Paris, Gallimard, 1982.

36. Mathiez, "La politique de Robespierre et le 9 thermidor expliqués par Buonarroti », AHRF, 1910, texte réédité dans La corruption parlementaire sous la terreur, Paris, Colin, 1927, puis dans Études sur Robespierre, Paris, 1958. Bronterre O’Brien, un des dirigeants du chartisme, publia : La vie et le caractère de M. Robespierre où l'on prouve par des faits et des arguments que ce personnage si calomnié fut l'un des plus grands hommes et l'un des réformateurs les plus purs et les plus éclairés qui aient jamais existé dans le monde, Londres, 1837. 
37. Une réédition en espagnol du livre d'ArthurRosenberg, Demokratie und Sozialismus, Berlin, 1937, est en préparation, préface d'Antoni Domenech (que je remercie pour ces informations) à paraître à Barcelone. Sur l'influence des travaux de Mathiez sur la gauche révolutionnaire allemande et italienne, voir Hedwig Hintze, Staatseinheit-Föderalismus im alten Frankreich und in der Revolution, Berlin/Leipzig, (1928), 1989, historienne allemande qui a rencontré Mathiez.

38. Charles A.Beard, Economic Origins of Jeffersonian Democracy (1915), New York, Free Press, 1965.

39. Horacio Sanguinetti, Robespierre. Esquema de biografia politica, Barcelone, 1970 ; Robespierre, La razon del pueblo, textes choisis, traduits et introduits par H. Sanguinetti, Buenos

Aires, 2003.

40. Mathiez, La Réaction thermidorienne, Paris, Colin, 1929, avertissement, p. VII-VIII.

\section{RÉSUMÉS}

Albert Mathiez, le grand historien de la Révolution française, n'a pas été reconnu, y compris dans la Société des études robespierristes qu'il fonda en 1907, comme l'un des premiers critiques lucides - et de gauche - de la Révolution bolchevik. Son bref passage au Parti communiste de 1920 à 1922, témoigne de son refus de dissocier socialisme et démocratie. En 1930, il devint une cible de l'historiographie stalinienne, qui condamna l'historien Mathiez, mais aussi « le jacobinisme » comme des adversaires de la « révolution prolétarienne ». Dans sa défense menée de 1930 jusqu'à sa mort en 1932, Mathiez jeta les pierres précieuses d'une critique lucide du phénomène stalinien à sa naissance.

Albert Mathiez, the great historian of the French Revolution was not recognized, nor the Société des études Robespierristes he founded in 1907, as one of the first lucid critiques - and of the Left of the Bolshevik Revolution. His brief period in the Communist Party from 1920 to 1922 attests to his refusal to separate socialism and democracy. In 1930, he became a target of Stalinist historiography, which condemned the historian Mathiez, but also « jacobinism » as adversaries of the proletarian revolution. In his defense conducted in 1930 until his death in 1932, Mathiez mounted a lucid critique of the phenomenon of Stalinism at its birth.

\section{INDEX}

Mots-clés : souveraineté populaire, démocratie, socialisme, totalitarisme, stalinisme, méthode historique

\section{AUTEUR \\ FLORENCE GAUTHIER}

Université Paris 7-Denis Diderot 6 rue de Lancry 75010 Paris gauthierflore@orange.fr 